\title{
Constance Scharff
}

\section{Trällern und Tirilieren.}

Biologische Parallelen zwischen menschlicher Sprache und Vogelgesang

In: Abecedarium der Sprache / Constanze Fröhlich, Martin Grötschel, Wolfgang Klein (Hg.). ISBN: 978-3-86599-416-5. - Berlin: Kulturverlag Kadmos, 2019. S. 205-213 


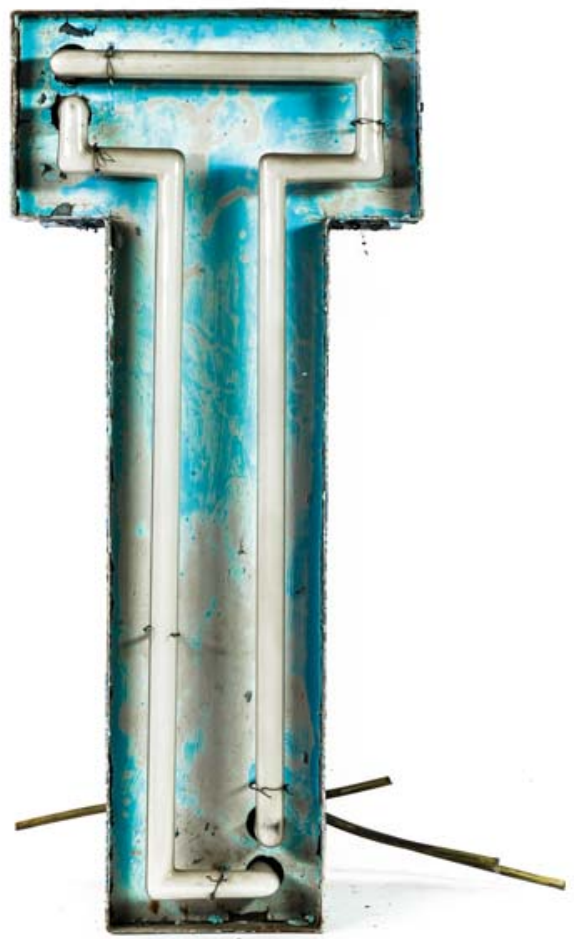


T-Berliner Metallhütten- und Halbzeug-Werke, Berlin Adlershof 


\section{Trällern und Tirilieren. Biologische Parallelen zwischen menschlicher Sprache und Vogelgesang}

Constance Scharff

Nicht nur die Nachtigall tiriliert, auch Amsel, Drossel, Fink und Star sowie die anderen über viertausend Singvogelarten der Welt sind begnadete Trällerer. Ihre Melodien beschwingen und faszinieren uns [ $\rightarrow$ Wonnig lallen]. Vielleicht können wir von den Vögeln sogar etwas darüber erfahren, wie der Mensch zu seiner Sprache gekommen ist? Vor über zweitausend Jahren bemerkte Aristoteles in seinen Zoologischen Schriften bereits erstaunliche Parallelen zwischen dem Vogelgesang und der menschlichen Sprache. Auch Darwin schrieb 1871, dass der Gesang der Vögel in verschiedenen Aspekten die beste Analogie zur Sprache sei (»The sounds uttered by birds offer in several respects the nearest analogy to language.« Darwin 1871: 55).

\section{Sprache und Vogelgesang}

Aristoteles beobachtete, dass junge Singvögel ihren Gesang von erwachsenen Vorbildern erlernen. Wie erstaunlich dieser Prozess den ersten zwei Jahren unseres Lebens ähnelt, in denen wir so viel Zeit damit verbringen, das Sprechen zu meistern, hat die moderne Forschung gezeigt: Das monologische Lallen des Säuglings wird abgelöst vom Babybrabbeln, aus dem sich mehr und mehr Silben entwickeln und dann das erste Wort, das große Ereignis des ersten Lebensjahrs. Danach entstehen einfache Konstruktionen, »mehr Keks «, und schließlich ganze Sätze. Ähnlich durchlaufen auch Singvögel verschiedene Meilensteine, bis sie das Singen perfektionie- 
ren: Nach den ersten Lauten im Nest, den Bettelrufen nach Futter, beginnt eine Phase, in der die oft erst gerade flügge gewordenen Jungvögel ohne Punkt und Komma brabbeln. Schon bald folgen die ersten Gesangssilben, die noch wirr durcheinander gesungen werden. Zunehmend kommt jedoch auch bei den Vögeln Ordnung und Syntax dazu und am Ende der Lernphase, wenn der Vogel erwachsen ist, hat er sein Lied gemeistert.

Sowohl für Menschen als auch für Singvögel ist der soziale Bezug während dieses Prozesses enorm wichtig. Je mehr mit Babys und Kleinkindern gesprochen wird, umso besser und schneller können sie die Sprachlaute nachahmen und benutzen. Dabei spielt der lebendige Austausch, die Kommunikation mit den Bezugspersonen eine Schlüsselrolle. Dies gilt auch für Singvögel. Sie lernen in der Interaktion mit erwachsenen Artgenossen viel besser und schneller, als wenn ihnen die zu erlernenden Gesänge nur vom Tonband vorgespielt werden. Und wer hätte gedacht, dass nicht nur Menschen ihre Sprache mit Gesten begleiten? Auch viele Singvögel koordinieren ihren Gesang mit Körperbewegungen, ein Thema unserer aktuellen Forschung (Ullrich/Norton/Scharff 2016).

\section{Gehirn, Sprache und Vogelgesang}

Weitere Parallelen zwischen Mensch und Federvieh betreffen die biologischen Mechanismen, die das Sprechenlernen bei Menschen und das Singenlernen bei Vögeln ermöglichen. Als Kinder eignen wir uns in der Regel ohne Mühe und akzentfrei eine oder mehrere Sprachen unserer sozialen Umgebung an. Singvögel sind in ihrer Jugend ebenfalls in der Lage, ihren arteigenen Gesang zu erlernen, manchmal sogar den Gesang einer anderen Art. Später, nach der Pubertät, können wir Menschen zwar weitere Sprachen erlernen, doch den meisten von uns gelingt dies nicht akzentfrei. Unsere Muttersprache drückt ihren Stempel auch auf Fremdsprachen, indem sie Rhythmus, Melodie und Aussprache beeinflusst. Laute, die in der eigenen Sprache nicht vorkommen, sind für Erwachsene meist schlecht zu meistern: Deutschen Muttersprachlern rollt 
das Spanische $r$ in ferrocarril nicht gut von den Lippen und sie tun sich oft schwer mit dem englischen th in the, these, there. Auch Singvögel lernen ihren Gesang am besten in einer begrenzten, der sogenannten sensiblen Jugendphase. Nach der Pubertät und dem damit veränderten hormonellen Milieu funktioniert das Nachahmen ihres erwachsenen Gesangstutors nur noch eingeschränkt.

Obwohl der letzte gemeinsame Vorfahre von Singvögeln und Menschen vor mehr als 300 Millionen Jahren gelebt hat, weisen sogar die Schaltkreise im Gehirn, die Menschen das Sprechen und Singvögeln das Tirilieren ermöglichen, erstaunliche Ähnlichkeiten auf. So ist eine Gehirnhemisphäre für die Sprachfähigkeit dominant, bei den meisten Menschen ist es die linke. Aus diesem Grunde beeinträchtigen Schlaganfälle in der linken Gehirnhälfte die Sprache typischerweise viel mehr als solche auf der rechten Seite. Bei Singvögeln dominiert ebenfalls eine Gehirnhälfte den Gesang. Welche die dominante Hälfte ist, variiert von Art zu Art. Bei Zebrafinken dominiert rechts, bei Kanarienvögeln links. Menschen und Singvögel ähneln einander frappierenderweise auch mehr als Menschen und Menschenaffen in der Art, wie bestimmte Gehirnregionen miteinander verbunden sind.

\section{Sprachgene?}

Schließlich gibt es sogar erstaunliche Parallelen bei Genen, die sowohl für die menschliche Sprache als auch für das Singen von Vögeln wichtig sind. Nun überrascht es zunächst vielleicht, dass Gene überhaupt für Sprache relevant sein sollen. Da das Sprechen erlernt werden muss und "genetisch" und »erlernt « fälschlicherweise oft als Gegensätze verstanden werden, ist die Auffassung verbreitet, dass Sprache keine genetische Komponente hat. Dies ist aber nicht der Fall. Gene beeinflussen alles Leben und deswegen auch das Lernen. Ob es allerdings Gene gibt, die eine spezielle Rolle für das Sprachlernen des Menschen spielen, wurde lange angezweifelt. Das lag daran, dass alle bekannten, durch Mutationen in Genen verursachten Krankheiten (»Erbkrank- 
heiten «), die die Sprachfähigkeit einschränkten, auch andere mentale Fähigkeiten beeinträchtigten. Deswegen war es eine große Überraschung, als 2001 in einer englischen Sprachheilschule bei mehreren Kindern einer verzweigten Familie ohne geistige Behinderung eine Mutation im sogenannten FOXP2Gen entdeckt wurde. Die Kinder nuschelten so stark, dass sie kaum zu verstehen waren. Genauere Untersuchungen zeigten, dass auch bei den Eltern und Großeltern ähnliche Artikulationsprobleme vorkamen, die ebenfalls durch die FOXP2-Mutation im Erbgut bedingt waren. In den darauffolgenden Jahren konnten klinische Genetiker zusammen mit Phoniatriespezialisten weltweit noch weitere Patienten mit demselben spezifischen Sprachdefizit identifizieren, bei denen ebenfalls eine Mutation im FOXP2-Gen zugrunde lag. Sie konnten auch zeigen, dass bei den Betroffenen weder die Hörfähigkeit eingeschränkt war noch eine allgemeine Muskel- oder Koordinationsschwäche existierte.

Diese Entdeckung führte zunächst zu großem Enthusiasmus bei denjenigen Linguisten, die schon seit langem postulierten, dass die menschliche Sprachfähigkeit auf einem besonderen, angeborenen Sprachinstinkt beruhe; sie argumentierten, dass ein Sprachinstinkt der Grund sei, warum trotz großer Unterschiede zwischen den Sprachen der Welt dennoch allen Sprachen gewisse universale linguistische Prinzipien gemein seien. Auch wenn diese Idee der »universellen Grammatik « bei Experten sehr umstritten war, trug sie zur Geburt des Mythos von FOXP2 als Sprachgen bei. Und da Sprache ja Kopfsache ist, wurde die Aktivität des FOXP2-Gens auch alsbald im Gehirn nachgewiesen sowie eine spezifisch menschliche Version des Gens bei Menschen entdeckt. Die Theorien, wie FOXP2 die Evolution unseres Gehirns verändert und damit die Evolution der Sprache hervorgebracht haben könnte, überschlugen sich.

Doch schon bald schlug der Enthusiasmus ins Gegenteil um, als sich herausstellte, dass die spezifisch menschliche Version des FOXP2-Gens nur ein Tausendstel der im Gen enthaltenen Information betrifft und dass alle Wirbeltiere 
extrem ähnliche FOXP2-Gene in ihrem Erbgut tragen. Da Fische, Vögel, Mäuse und Ratten normalerweise nicht sprechen, war die Enttäuschung groß. Zudem wurde bekannt, dass das FOXP2-Gen nicht nur im Gehirn eine Rolle spielt, sondern auch in vielen anderen Organen, unter anderem Herz, Lunge und Darm.

Doch war die Enttäuschung gerechtfertigt? Um dies zu beleuchten, muss man die Funktion von Genen etwas genauer betrachten. Gene sind die Bauanleitung für Proteine, die Bausteine, aus denen Zellen und Gewebe zusammengesetzt sind. Genauso wie man aus Ziegeln sowohl Kathedralen als auch Supermärkte oder Einfamilienhäuser bauen kann, gibt es Proteine, die für Gehirn, Herz und Lunge gleichermaßen wichtig sind. Die Tatsache, dass FOXP2 also nicht nur im Gehirn gebraucht wird, schließt nicht aus, dass es für die Sprache besonders relevant sein kann.

Aber widerspricht die Tatsache, dass das FOXP2-Gen auch bei so vielen Tieren existiert, nicht der Möglichkeit, dass es für die menschliche Sprache spezifisch wichtig sein soll? Auch dies muss verneint werden. Man kann sich das Zusammenspiel von Proteinen auch ein bisschen wie Kochen vorstellen. Mit unterschiedlichen Zutaten und Kochmethoden können sehr verschiedene, doch jeweils einzigartige Gerichte entstehen. Eine Gruppe von Proteinen kann also beispielsweise bei Fisch und Mensch in fast unveränderter Form vorliegen, aber die unterschiedliche Art ihres Zusammenwirkens und der Einfluss zusätzlicher Faktoren können beim Fisch zur Ausbildung von Flossen führen und beim Menschen zur Entwicklung von Gliedmaßen.

Kommen wir zurück zum FOXP2-Gen und dessen besonderer Funktion für die Sprache. Welche Rolle spielt es in der Küche, in der die Sprachfähigkeit "gebacken « wird? Ist es wichtig für eine zentrale, sprachessenzielle Komponente wie die Hefe für den Hefezopf? Oder ist FOXP2 eher eine Zutat, die auch gebraucht wird, aber weniger spezifisch ist, wie die Prise Salz? Oder ist das FOXP2-Gen gar so wenig spezifisch wie der Strom, mit dem nicht nur der Backofen betrieben wird, sondern auch zig andere Geräte? 
Hier schlagen wir den Bogen zurück zu den Singvögeln. Wenn FOXP2 speziell für die Fähigkeit wichtig wäre, als Baby die Sprachlaute der Umwelt zu identifizieren und dann im Laufe des ersten Lebensjahres aus den ungeformten ersten Lalllauten nach und nach durch Nachahmung die Muttersprache zu entwickeln, dann könnte FOXP2 bei Singvögeln vielleicht eine ähnliche Funktion beim Singenlernen haben? Ermutigt durch die Tatsache, dass FOXP2-Aktivität bei Zebrafinken ausgerechnet in der Gehirnregion nachgewiesen werden konnte, die bereits als Gesangslernzentrum bekannt war, haben wir in einem Experiment bei brabbelnden Jungvögeln die Aktivität des FOXP2-Gens in dieser Region reduziert. Und tatsächlich führte dies dazu, dass die jungen Vögel den Gesang ihrer erwachsenen Vorsänger, ihrer »Tutoren «, danach viel schlechter nachahmten, als dies normalerweise der Fall ist. Erstaunlicherweise ähnelten die Gesangsstörungen sogar den Sprachstörungen der menschlichen Patienten mit einer FOXP2-Mutation: Die Zebrafinken hatten ein kleineres Gesangsrepertoire als ihre Tutoren, analog zu den kürzeren, einfacheren Sätzen, die für Patienten mit FOXP2-Mutationen charakteristisch sind. Zudem war die »Aussprache« der Gesangssilben bei den behandelten Zebrafinken auch besonders nuschelig (Haesler et al. 2007). Diese Befunde unterstreichen eindrucksvoll, dass eine verminderte Menge des FOXP2Proteins sowohl bei Menschen als auch bei Singvögeln das Erlernen von Kommunikationslauten beeinträchtigt.

Helfen uns diese Resultate nun zu verstehen, welche Bedeutung dieses Gen für die Sprache hat? Welche Funktion spielt das Gen in Gehirnzellen? Das FOXP2-Gen gehört zu den sogenannten Transkriptionsfaktoren, die regulieren, ob andere Gene aktiv »abgelesen « und dadurch in Proteine umgeschrieben werden können oder ob sie inaktiv sind. Ein einziger Transkriptionsfaktor kann hunderte anderer Gene regulieren. Im Falle von FOXP2 sind eine ganze Anzahl dieser sogenannten FOXP2-Zielgene bereits identifiziert worden durch Forschung an humanen Zellen und Geweben, an Mäusen und Singvögeln. Wir wissen auch, dass manche Zielgene wichtig für die Informationsübertragung zwischen 
Nervenzellen im Gehirn sind, die das Lernen von besonders schnellen Bewegungen ermöglichen. Veränderte FOXP2Proteinmengen beeinträchtigen beim Menschen die schnelle deutliche Aussprache, bei Mäusen schnelles, trittsicheres Rennen und bei Zebrafinken schnelles, präzises Singen. Diese Ergebnisse bieten Erklärungsansätze, warum FOXP2-Mutationen beim Menschen zum Nuscheln führen. Sie erklären aber noch nicht, warum Patienten auch Probleme mit dem Verständnis komplexer grammatischer Satzkonstruktionen haben, wie »der Bleistift, der auf dem grünen Tisch liegt, ist rot«. Die Ergebnisse erklären auch nicht, warum Menschen sprechen und Menschenaffen nicht. Um diesen Geheimnissen auf die Spur zu kommen, haben Forscher die ganz leicht abweichende Version des menschlichen FOXP2-Gens experimentell in Mäuse eingeschleust. Die Tatsache, dass Mäuse daraufhin zwar nicht zu sprechen begannen, aber gewisse Gehirnzellen anders funktionierten und auch bestimmte Lautäußerungen und das Lernverhalten verändert waren, zeigt sehr eindrücklich, dass FOXP2 nicht »das Sprachgen « ist, kein Zauberstab, mit dessen Hilfe Sprache plötzlich aus dem Nichts gezaubert werden kann. Vielmehr ist es ein Gen, das durch die Regulation vieler anderer Gene zur normalen Entwicklung des Gehirns beiträgt, vor allem für bestimmte Gehirnstrukturen, die Sinneseindrücke wie Hören und Fühlen in Bewegungen umsetzen. Versuche mit Singvögeln zeigen auch, dass FOXP2 auch nach der Entwicklung, also im erwachsenen Tier gebraucht wird, damit Zebrafinken ihren Gesang richtig einsetzen. Richtig einsetzen? Warum singen Vögel eigentlich?

\section{Funktion von Sprache und Vogelgesang}

Sehr viele Experimente können zeigen, dass es zwei Funktionen von Gesang gibt: die Balz und die Revierverteidigung. Der Gesang eines Vogels kann dem potenziellen Paarungspartner sehr viel Aufschluss über seine "genetische Qualität « mitteilen: Wenn zum Beispiel ein Nachtigallmännchen ein sehr großes, variationsreiches Gesangsrepertoire erlernt hat, 
das es viele Nachtstunden lang laut schmettert, dann hat dieser Nachtigall-Caruso bei den Weibchen bessere Chancen als ein Männchen mit kleinerem Repertoire, dem schon nach kurzer Zeit die Puste ausgeht. Bei vielen Vögeln wird der Gesang auch zum Ausloten von Rivalitäten eingesetzt. Reviere müssen erworben und verteidigt werden, und wer der Stärkere ist, zeigt sich ebenfalls oft im Gesang. Ein klassisches Experiment mit Meisen bewies beispielsweise, dass Reviere, aus denen Meisengesang aus Lautsprechern schallte, von anderen Männchen gemieden wurden, dass also der Gesang allein schon abschreckende Wirkung für Rivalen hat. Und Weibchen hören bei Revierauseinandersetzungen zwischen Männchen oft zu und wählen dann den Gewinner eines Sängerstreits öfter als Paarungspartner als den Verlierer.

Auch wir Menschen setzen unsere Sprache zum Flirten und zum Streiten ein, aber zusätzlich können wir damit, um Wilhelm von Humboldt zu zitieren »von endlichen Mitteln einen unendlichen Gebrauch machen «(Humboldt 1836: 106). Wir können unser endliches artspezifisches Lautrepertoire zu Silben formen, diese zu unendlich vielen Worten verbinden und schließlich durch deren Kombination unendlich viele Sätze schöpfen. Wir können so unser Denken in Laute (oder Gebärden) umwandeln, damit die Vergangenheit beschreiben oder über die Zukunft philosophieren, wir haben die Wahl, mit Sprache wahrhaftig zu sein oder zu lügen $[\rightarrow$ Flunkern]. Die Regeln der Sprache geben uns die Fähigkeit, winzige Lautunterschiede richtig zu interpretieren: »Der Mann hat die Frau umgebracht « oder »Den Mann hat die Frau umgebracht «. Tieren werden derartige Fähigkeiten im Großen und Ganzen abgesprochen, ohne dass wir dafür sehr gute Beweise hätten. Zugegeben, bisher gibt es keine Google-Übersetzungsfunktion, mit der wir erfahren können, ob Tiere sich mehr mitteilen, als dass ein Feind im Anmarsch ist, dass sie bereit sind, sich zu paaren, oder dass sie einem Rivalen den Kampf androhen. Es lohnt sich aber, darüber nachzudenken, welche Experimente zeigen können, ob beim Trällern und Tirilieren der Vögel etwas mitgeteilt wird, was wir noch nicht entdeckt haben. Ob wir die Vögel dann 


\section{allerdings besser verständen? Wittgenstein jedenfalls war skeptisch: »Wenn ein Löwe sprechen könnte, wir könnten ihn nicht verstehen « (Wittgenstein 1953: 568).}

\section{Literatur}

Aristoteles (2013): Historia Animalium. Zoologische Schriften. Übers., eingel. und komm. v. Stephan Zierlein. Berlin: Akademie-Verlag. (Die oben wiedergegebenen Ausführungen in 504a35-504b3 sowie 536b.)

Darwin, Charles (1871): The Descent of Man and Selection in Relation to Sex (First ed.). London: John Murray.

Haesler, Sebastian/Rochefort, Christelle/Georgi, Benjamin/Licznerski, Pawel/ Osten, Pavel/Scharff, Constance (2007): »Incomplete and inaccurate vocal imitation after knockdown of FOXP2 in songbird basal ganglia nucleus Area X«. In: PLoS Biology. 5 (12). S. e321.

Humboldt, Wilhelm von (1836): Über die Verschiedenheit des menschlichen Sprachbaues und ihren Einfluß auf die geistige Entwickelung des Menschengeschlechts. Berlin: Königliche Akademie der Wissenschaften.

Ullrich, Robert/Norton, Philipp/Scharff, Constance (2016): »Waltzing Taenioygia: integration of courtship song and dance in the domesticated zebra finch «. In: Animal Behavior. 112. S. 285-300.

Wittgenstein, Ludwig [1953] (1984): Philosophische Untersuchungen. In: Ders.: Werkausgabe. Bd. 1. Frankfurt am Main: Suhrkamp. 
